Revue d'histoire de l'Amérique française

REVUE D.HISTOIRE DE L'AMÉRIQUE FRANÇAISE

\title{
Sur un démarrage raté : industrie laitière et co-intégration au Saguenay (1880-1940)
}

\section{Gérard Bouchard}

Volume 45, numéro 1, été 1991

URI : https://id.erudit.org/iderudit/304948ar

DOI : https://doi.org/10.7202/304948ar

Aller au sommaire du numéro

\section{Éditeur(s)}

Institut d'histoire de l'Amérique française

\section{ISSN}

0035-2357 (imprimé)

1492-1383 (numérique)

Découvrir la revue

\section{Citer cet article}

Bouchard, G. (1991). Sur un démarrage raté : industrie laitière et co-intégration au Saguenay (1880-1940). Revue d'histoire de l'Amérique française, 45(1),

73-100. https://doi.org/10.7202/304948ar
Résumé de l'article

À partir du cas du Saguenay, on explore l'hypothèse suivante : ce sont des facteurs sociaux et culturels qui ont retardé l'essor de l'industrie laitière et la restructuration de l'économie agricole, contrariant les conditions favorables pourtant présentes dès la fin du siècle dernier. L'explication réside peut-être dans le modèle de la co-intégration. 


\title{
SUR UN DÉMARRAGE RATÉ: INDUSTRIE LAITIÈRE ET CO-INTÉGRATION AU SAGUENAY (1880-1940)
}

\author{
GÉRARD BOUCHARD ${ }^{1}$ \\ Centre interuniversitaire SOREP \\ Université du Québec à Chicoutimi
}

\section{RÉSUMÉ}

À partir du cas du Saguenay, on explore l'hypothèse suivante: ce sont des facteurs sociaux et culturels qui ont retardé l'essor de l'industrie laitière et la restructuration de l'économie agricole, contrariant les conditions favorables pourtant présentes dès la fin du siècle dernier. L'explication réside peut-être dans le modèle de la co-intégration.

\section{ABSTRACT}

This study of agriculture in the Saguenay region examines the following hypothesis: social and cultural factors delayed the progress of the dairy industry and the restructuration of the farm economy though the material conditions were there as early as the end of the last century. "Co-integration» may be the key to this phenomenon.

Depuis plus d'une trentaine d'années, l'historiographie du Québec rural a été le lieu d'une importante discussion sur les supposés «retards» de l'économie agricole canadienne-française. En rapport avec ce débat, une attention particulière doit être accordée aux mutations ayant donné lieu à l'essor de l'industrie laitière dans les dernières décennies du XIX ${ }^{\mathrm{e}}$ siècle. Cet épisode de l'histoire rurale québécoise est remarquable à cause des changements structurels à long terme qu'il a entraînés et, plus encore peut-être, à cause de certaines difficultés qu'il pose à l'analyse. Après un départ encourageant qui

1 Les travaux qui ont conduit à la rédaction de cet article ont pu être réalisés grâce à l'assistance financière du Conseil de recherches en sciences humaines du Canada, du Fonds FCAR (Québec) et de l'Université du Québec à Chicoutimi. L'auteur remercie Régis Thibeault (collecte et validation de données), Lynda Villeneuve (cartographie), Carole Roy et Judith Goulet (traitement de texte), Normand Perron et Marc Saint-Hilaire (lecture critique du manuscrit) pour leur précieuse collaboration. 
annonçait une conversion rapide et en profondeur à l'économie capitaliste, l'industrie laitière a montré d'importants signes de freinage pendant le premier tiers du $\mathrm{XX}^{\mathrm{e}}$ siècle. L'explication de ce phénomène, à laquelle le présent article est consacré, oblige à déborder les paramètres strictement techniques ou économiques de l'agriculture. Nous suggérons l'hypothèse suivante: les conditions favorables à un développement rapide de l'industrie laitière et à une restructuration complète de l'économie agricole se trouvaient réunies dans la majorité des régions du Québec dès la fin du siècle dernier; ce sont des facteurs sociaux et culturels qui ont fait échec à une exploitation complète de ces conditions, décalant ainsi de quelques décennies le calendrier des mutations structurelles.

Cette hypothèse sera explorée en prenant à témoin le cas du Saguenay, justement l'une des régions les plus défavorisées objectivement, du moins en apparence, à cause de l'éloignement ${ }^{2}$. Nous nous limiterons toutefois à un survol des principaux éléments du dossier à partir de repères statistiques et qualitatifs parmi les plus significatifs, de manière à faire ressortir la cohésion et la pertinence du cadre explicatif proposé ${ }^{3}$. Les principales sources utilisées consistent dans les journaux régionaux publiés entre 1882 et 1930 , divers corpus d'histoire orale constitués depuis 1970 par l'auteur (sauf celui des Mémoires d'anciens conservés aux Archives nationales du Québec à Chicoutimi [à l'avenir, ANQC]), les recensements canadiens et certains fonds d'archives plus spécifiques qui seront mentionnés en cours de route. Enfin, nous nous sommes aussi appuyé sur le mémoire de maitrise de Régis Thibeault ${ }^{4}$ et sur quelques travaux de Normand Perron ${ }^{5}$.

2 À plus de $200 \mathrm{~km}$ au sud, et à une douzaine d'heures de train, Québec était en 1900 la ville la plus proche.

3 L'ensemble du dossier fait par ailleurs l'objet d'une recherche doctorale inscrite récemment au département d'histoire de l'Université Laval par Régis Thibeault, chercheur à SOREP.

$4 \quad$ R. Thibeault, Les transformations de l'agriculture au Saguenay, 1852-1971, thèse de M.A. (études régionales), Université du Québec à Chicoutimi, 1988, 256 p.

5 N. Perron, «Genèse des activités laitières, 1850-1960», N. Séguin, dir., Agriculture et colonisation au Québec (Montréal, Boréal Express, 1980), 113-140; «Une agriculture naissante» (chapitre 5: 155-184), «Une agriculture fragile» (chapitre 8: 255-284), "Coopératives et rationalisation de l'agriculture depuis 1930» (chapitre 11: 385-416), C. Girard, N. Perron, Histoire du Saguenay-Lac-Saint-Jean (Québec, Institut québécois de recherche sur la culture, 1989), 665 p.; «L'évolution des institutions coopératives agricoles au Saguenay et au Québec (1909-1950)», soumis pour publication (1990). Par ailleurs, les données des recensements canadiens ont été soumises à diverses opérations de critique et de validation qui ne seront pas rappelées ici. Voir R. Thibeault, «Les unités de mesure dans les documents officiels du dixneuvième siècle au Bas-Canada et au Québec», Revue d'histoire de l'Amérique française, 43,2 (automne 1989): 221-232; R. Thibeault, G. Bouchard, Données décennales relatives aux 


\section{1 - UNE PROBLÉMATIQUE DE LA CROISSANCE ET DU CAPITALISME AGRAIRE}

On connaît assez, du moins dans les grandes lignes, les principales causes externes qui, dans le dernier tiers du $\mathrm{XIX}^{\mathrm{e}}$ siècle, ont poussé un très grand nombre d'agriculteurs québécois à se tourner vers l'élevage laitier en vue de la production du fromage et du beurre. L'existence d'un marché international, les pressions gouvernementales et le déclin de l'économie céréalière viennent en tête parmi les facteurs le plus souvent mentionnés ${ }^{6}$. On connaît sans doute moins les conditions internes et les modalités locales ou régionales de cette conversion. C'est sur cette dynamique interne que le présent essai veut attirer l'attention en prenant pour cible ce qu'on pourrait appeler les structures sociales de l'industrie laitière. Plus précisément, il s'agit d'expliquer à la fois les éléments de freinage au début du $\mathrm{XX}^{\mathrm{e}}$ siècle et les conditions de leur élimination surtout à partir de 1935-1940.

\section{A - Sur le sous-développement de l'agriculture}

Depuis longtemps et de diverses façons, l'agriculture du Québec francophone a été mise en procès. Il est impossible de présenter ici toutes les pièces de ce dossier ${ }^{7}$. Essayons d'aller à l'essentiel. On peut dire que la question du sous-développement de l'agriculture a été traitée de deux façons. En premier lieu, selon la problématique du capitalisme agraire. Contrairement à ce qui s'est passé dans plusieurs régions des États-Unis et du Canada anglais, l'idéal de la propriété familiale, associée au conservatisme et à la stagnation, aurait longtemps prédominé dans l'agriculture québécoise (entendons ici: francophone $)^{8}$. B. Bernier y voit le double effet d'une stratégie des élites politiques qui trouvaient intérêt à maintenir une agriculture familiale, et d'une monopolisation du capital industriel qui décourageait l'investissement dans le secteur agricole ${ }^{9}$. Après d'autres, M. Morisset a

superficies cultivées et améliorées dans les fermes du Saguenay, par paroisse (1852-1971) (SOREP, Document no II-C-147, 1989), 57 p.; G. Bouchard, R. Thibeault, «L'économie agraire et la reproduction sociale dans les campagnes saguenayennes (1852-1971)», Histoire sociale/ Social History, 18,36 (novembre 1985): 237-257.

6 J. Hamelin, Y. Roby, Histoire économique du Québec: 1851-1896 (Montréal, Editions Fides, coll. «Histoire économique et sociale du Canada français», 1971), 436 p.; N. Perron, "Genèse des activités laitières...», loc. cit.; F. Létourneau, Histoire de l'agriculture (s.l., s.c., 1968), 398 p.

7 On en trouvera un bon échantillon dans le collectif réalisé sous la direction de N. Séguin, Agriculture et colonisation au Québec (Montréal, Boréal Express, 1980), 220 p.

8 Pour un contre-exemple, voir l'analyse que fait P. Voisy du développement agricole dans le sud de l'Alberta au tournant du siècle. P. Voisy, VULCAN. The Making of a Prairie Community (Toronto, University of Toronto Press, 1988), 341 p. 73-91.

9 B. Bernier «La pénétration du capitalisme dans l'agriculture», N. Séguin, dir., op. cit., 
proposé la thèse de la régression du salariat agricole au profit de la main-d'œuvre familiale ${ }^{10}$. Rendus déficitaires par l'exploitation capitaliste, les agriculteurs auraient réagi en misant sur un sur-travail non rémunéré fourni par la famille ${ }^{11}$. D'autres auteurs accusent aussi l'accès facile à la propriété dû à l'abondance des terres disponibles, la structure des mentalités ou, comme B. Jean et O. Stanek, les séquelles d'un vieux projet de société coloniale ${ }^{12}$.

Dans la deuxième perspective, les problèmes de développement agricole sont simplement définis en référence à la productivité: qu'estce qui a rendu l'agriculteur québécois moins productif? Ici on met en cause tour à tour - ou conjointement - l'éloignement et l'absence de marchés ${ }^{13}$, le système agro-forestier ${ }^{14}$, des facteurs conjoncturels et structurels liés soit à la concurrence et à l'environnement nordaméricain $^{15}$, soit à la faible capacité d'investissement des exploitants ${ }^{16}$, enfin des facteurs culturels qui auraient entraîné des comportements réactionnaires et rendu le paysan incapable de s'adapter aux nouvelles situations ${ }^{17}$.

Dans un cas comme dans l'autre, soit parce qu'elles s'en tiennent à des facteurs externes, soit parce qu'elles privilégient un facteur en particulier, les démarches qui viennent d'être mentionnées ne portent pas assez attention à un facteur global qui consiste dans les structures de la société paysanne et la dynamique de sa reproduction. Notre

10 M. Morisset, «Éléments pour une analyse matérialiste de l'agriculture au Québec», Les Cahiers du socialisme, 7 (1980): 73-95.

11 Selon l'auteur, la thèse ne vaudrait toutefois pas pour la période allant de 1966 à nos jours, durant laquelle on verrait apparaitre un rapport de classe dans l'agriculture québécoise.

12 B. Jean et $\mathrm{O}$. Stanek, «L'exploitation agricole familiale au Québec», H. Lamarche, dir., Les capacités d'adaptation de l'exploitation agricole: comparaison internationale (Nanterre, CNRS, Université de Paris X, 1987), 17-55.

13 Parmi d'autres: C. Lemelin, «Social Impact of Industrialization on Agriculture in the Province of Quebec», Culture, 14,1 (mars et juin 1953): 34-46 et 157-169; J. McCallum, Unequal Beginning (Toronto, University of Toronto Press, 1980), $148 \mathrm{p}$.

14 N. Séguin, La conquête du sol au 19e siècle (Québec, Boréal Express, 1977), $295 \mathrm{p}$.

15 J.-P. Wampach, "Deux siècles de croissance agricole au Québec, 1760-1985», Recherches sociographiques, 29,2-3 (1988): 181-199.

16 A. Greer, Peasant, Lord, and Merchant: Rural Society in Three Quebec Parishes, 1740-1840 (Toronto, University of Toronto Press, 1985), 304 p; O. Stanek, «Dynamique de la structuration de l'espace agricole: l'exemple de l'Est du Québec des années trente», Histoire sociale/Social History, 20,39 (1987): 101-128.

17 Sur ce dernier point, la bibliographie est abondante. N. Casteran en a fait récemment une présentation utile. N. Casteran, «Les stratégies agricoles du paysan canadien-français de l'est ontarien (1870)», Revue d'histoire de l'Amérique française, 41,1 (été 1987): 23-51. Au chapitre des explications, il faut signaler aussi l'essai d'un géographe, R. De Koninck, construit autour des tensions qui naîtraient au coeur de la territorialité rurale. R. De Koninck, «Pourquoi les paysans? Interrogations sur la territorialité de l'agriculture familiale et notes sur le cas québécois», Cahiers de géographie du Québec, 28,73-74 (avril-sept. 1984): 261-274. 
tentative se porte précisément dans cette direction. Par ailleurs, s'agissant de l'agriculture québécoise des $\mathrm{XIX}^{\mathrm{e}}$ et $\mathrm{XX}^{\mathrm{e}}$ siècles, il n'y a pas d'inconvénient majeur à fusionner les deux perspectives évoquées plus haut dans la mesure où l'intégration poussée à l'économie de type capitaliste s'est par définition caractérisée par une recherche du profit par le biais d'une hausse de la productivité, celle-ci résultant d'une réorganisation en profondeur des structures de production. Il est utile toutefois de préciser ce qu'on entend ici par capitalisme agraire. Ce concept peut en effet être défini tour à tour ou simultanément par référence à: a) l'intégration poussée à un marché autre que local; b) la diffusion du salariat et l'installation d'un rapport de travail antagoniste; c) la disparition de l'emprise familiale sur l'exploitation (critère qui ne se confond qu'en partie avec le précédent); d) une orientation de gestion axée sur la rationalité du profit; e) un processus de spécialisation tendant à une exploitation maximale des facteurs de production. Du point de vue théorique, cette pluralité de critères pourrait alimenter un long débat. Nous nous laisserons guider ici par le pragmatisme, en faisant remarquer que, concrètement, la conversion au capitalisme agraire n'est souvent que partielle - l'histoire offre au demeurant peu d'exemples de types purs - et surtout que cette conversion, loin d'être brutale, prend ordinairement la forme d'une évolution lente. Comme il arrive fréquemment, on a plus affaire ici à un continuum qu'à une dualité exclusive ${ }^{18}$. En outre, la discussion théorique achoppe sur la difficulté de construire des indicateurs fiables et pertinents (par exemple, quand passe-t-on de la simple vente des surplus à la commercialisation généralisée puis au capitalisme «intégral»?). En tout état de cause, et pour des raisons qui tiennent à la fois à des contraintes de sources et à des problèmes de méthode, nous nous en remettrons dans les pages qui suivent aux seuls critères de la commercialisation, de la spécialisation et de la productivité.

\section{B - Un modèle explicatif: la co-intégration}

Les facteurs sociaux et culturels accusés ici d'avoir fait obstacle au développement du capitalisme agraire sont réunis dans ce que nous appelons la co-intégration. Ce modèle ayant déjà été présenté ailleurs ${ }^{19}$, il suffira d'en rappeler les principaux éléments. Il faut

18 Et dans cette mesure, la très riche controverse suscitée aux États-Unis par les travaux de J. T. Lemon et J. Henretta perd peut-être un peu de son intérêt. J. T. Lemon, The Best Poor Man's Country: a Geographical Study of Early Southeastern Pennsylvania (Baltimore, 1972); J. A. Henretta, «Families and Farms: Mentalité in Pre-Industrial America», William and Mary Quarterly, 3rd series, 35,1 (January 1978): 3-32.

19 G. Bouchard, «Co-intégration et reproduction de la société rurale. Pour un modèle saguenayen de la marginalité», Recherches sociographiques, 29,2-3 (1988): 283-310. 
d'abord écarter l'idée simpliste d'une corrélation entre tel facteur socio-culturel et tel comportement économique (ou tel signe de sousdéveloppement). Le problème est beaucoup plus complexe, mettant en cause l'ensemble d'un système social. Le modèle de la co-intégration vise précisément à caractériser la dynamique propre à la société rurale (dite aussi «locale» ou «marginale») et la nature des rapports qu'elle entretenait avec l'économie ou la société extra-régionale (ce que Marcel Mauss appelait la société globale et qu'on peut associer ici au capitalisme national et international). Il s'agit essentiellement de redéfinir, en les précisant, les modèles de l'auto-subsistance et de la marginalité, à partir de cinq idées-maîtresses:

1 - La société locale était animée par une dynamique de la reproduction centrée sur les solidarités familiales et communautaires. Cette dynamique visait à préserver un maximum d'indépendance ou d'autonomie collective par rapport à la société globale.

2 - La société locale parvenait à se perpétuer en tirant profit des sources d'emploi et de numéraire offertes par l'économie extrarégionale (travail saisonnier ou à temps partiel dans le secteur industriel, exportation des produits du travail domestique, etc). Cette stratégie se traduisait par la pluriactivité, caractéristique fondamentale de l'exploitation paysanne, qui fait référence à la fois au caractère mixte de l'agriculture comme telle et aux activités non agricoles.

3 - La pluriactivité était rendue possible par l'existence d'une maind'œuvre familiale nombreuse et relativement soumise, qui acceptait de se sacrifier pendant plusieurs années au profit des aînés. Ce «service familial» était partie intégrante d'une dynamique communautaire plus large ${ }^{20}$.

4 - L'ensemble des revenus d'appoint tirés des travaux «externes» (ou non agricoles) permettait à la société paysanne d'assurer son intégration et sa reproduction. En même temps, le travail et les produits à bon marché qu'elle fournissait au secteur industriel et à l'économie capitaliste en général contribuaient à la reproduction et à l'intégration de la société globale. Ainsi les deux entités ou systèmes sociaux assuraient leur survie et leur croissance respectives mais dans l'interdépendance: d'où le concept de cointégration. À l'échelle locale, la pluriactivité en était l'expression

20 G. Bouchard, «La dynamique communautaire et l'évolution des sociétés rurales québécoises aux $\mathrm{XIX}^{c}$ et $\mathrm{XX}^{c}$ siècles. Construction d'un modèle», Revue d'histoire de l'Amérique française, 40,1 (été 1986): 51-71. 
économique, et la dynamique communautaire son expression sociale.

5 - Il en résulte que, grâce aux liens établis avec le système économique et social de type moderne, la société rurale est parvenue à perpétuer pendant un temps ses traits les plus traditionnels (fécondité élevée, solidarité familiale, scolarisation rudimentaire, etc).

Ainsi, la co-intégration désigne un modèle social qui met en place un ensemble de rapports d'un type particulier avec le marché, et ce modèle n'est assimilable ni à l'auto-subsistance ni au capitalisme. Nous pensons que des rapports de ce genre se sont instaurés entre la société rurale saguenayenne et l'économie extra-régionale à partir des débuts du peuplement en 1840 jusqu'en 1935-1940 environ. Et nous faisons l'hypothèse que ces rapports ont engendré la stagnation de l'industrie laitière, dont le revenu était géré comme celui du travail forestier ou de la cueillette des bleuets: on y voyait un nouvel apport de numéraire parmi d'autres, modeste mais régulier et fiable, plutôt qu'une voie d'enrichissement sur laquelle il aurait été avisé de concentrer toutes ses ressources et faire reposer sa survie.

\section{2 - SUR UN DÉMARRAGE RATÉ}

Dans cette perspective, il convient d'abord d'établir clairement le fait de la stagnation de l'industrie laitière dans les campagnes saguenayennes en retraçant brièvement son évolution entre 1880 et 1940 1945.

\section{A - Un premier démarrage}

Entre les années 1880 et le début du $\mathrm{XX}^{\mathrm{e}}$ siècle, on assista au Saguenay à une série de transformations que plusieurs contemporains identifièrent à une véritable révolution dans la paysannerie. On sait qu'à l'origine, l'essor de l'industrie laitière a été favorisé par le déclin de l'économie céréalière au Québec, de même que par l'existence d'une forte demande à l'échelle nationale (reliée à l'urbanisation) et surtout internationale (essentiellement la Grande-Bretagne) ${ }^{21}$. D'autres facteurs ont contribué à cette mise en place, en particulier l'extension de l'écoumène agricole québécois consécutive au mouvement de colonisation, les conditions pédologiques et surtout climatiques des nouvelles régions et l'existence d'un régime quasi universel de petits exploitants-propriétaires, maîtres sur leur ferme. Au Saguenay, le

21 R. Duhaime, «Les exportations de produits laitiers, 1867 à 1955», L'Actualité économique, 23,2 (1957): 242-270. 
calendrier d'implantation des premières fromageries n'accuse guère qu'un décalage d'une dizaine d'années par rapport au mouvement provincial. En gros, la cartographie et la chronologie de cette implantation se modulent sur la marche du peuplement (Cartes 1, 2 et $3)$. Les premières fromageries étaient en exploitation dès 1881-1882 dans le Haut-Saguenay (à la Baie et à Chicoutimi) et dans le Sud-Est du Lac-Saint-Jean (Hébertville, bientôt suivie de Métabetchouan, Chambord, Roberval, etc) ${ }^{22}$. Quelques-unes de ces dernières créations sont remarquables car elles précédaient la construction du chemin de fer Québec-Lac-Saint-Jean, inauguré en 1888. Le fromage devait donc être acheminé par chemin de terre au port de Chicoutimi. En général, dans la plupart des paroisses ouvertes entre 1880 et 1940, il s'écoulait de 5 à 20 ans entre l'arrivée des premiers colons et l'ouverture de la première fabrique ${ }^{23}$. À la fin du siècle, la région comptait une soixantaine de fabriques (dont 6 beurreries seulement) réparties quasi également entre le Lac-Saint-Jean et les deux sous-régions de l'est. Durant les vingt années suivantes, ce nombre allait se multiplier par deux.

Parallèlement, on observait des changements importants dans la technologie, l'équipement et les productions agricoles. Entre 1890 et 1895 , des silos apparurent sur les fermes, en même temps que les hache-paille et les fameuses «boîtes à fermentation» (nombreuses mentions dans la presse locale). Le but visé était d'allonger la saison de lactation en donnant du fourrage aux vaches durant l'hiver. À la même époque, on notait plusieurs autres changements dans les procédés culturaux et l'outillage agraire ${ }^{24}$. C'était le temps des inventeurs, des «patenteux», qui à leur façon témoignent aussi de l'effervescence qui paraissait secouer le monde paysan ${ }^{25}$. Comme dans l'ensemble du Québec ${ }^{26}$, les productions subissaient aussi des mutations importantes. Le blé déclinait rapidement au profit de l'avoine, du foin, de la lentille, du trèfle et autres fourrages ${ }^{27}$. Quant au troupeau laitier, il est passé de 5 à 10 vaches par ferme dans l'ensemble de la région entre 1891 et 1921. Il est certain par ailleurs que le chemin de fer qui relia

22 Le Saguenay, 30 janvier et 10 août 1883; Le Progrès du Saguenay, 24 novembre 1892 et 15 mai 1919.

23 Tableau non reproduit ici. Voir G. Bouchard, R. Thibeault, Données sur l'évolution de l'industrie laitière dans la région du Saguenay (SOREP, Document no II-C-168, 1990), $10 \mathrm{p}$.

24 Ils sont rapportés dans G. Bouchard, «L'agriculture saguenayenne entre 1840 et 1950: l'évolution de la technologie», Revue d' histoire de l'Amérique française, 43,3 (hiver 1990): 353380.

25 L'un d'eux est dit avoir mis au point un moulin à battre mû par le vent (Le Progrès du Saguenay, 3 juin 1897, 5).

26 N. Perron, Genèse des activités..., loc. cit.

27 R. Thibeault, op. cit., chapitre IV. 


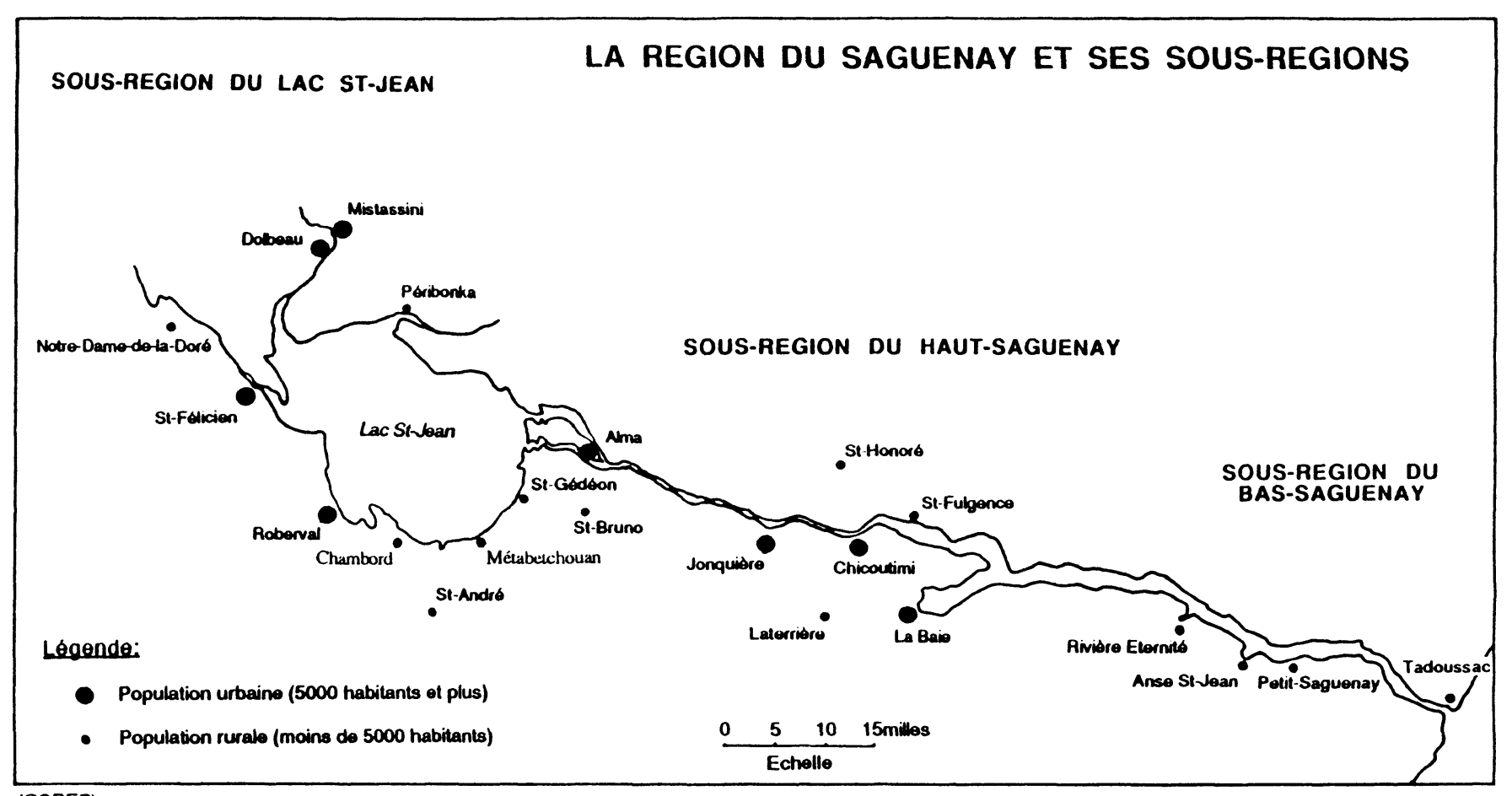




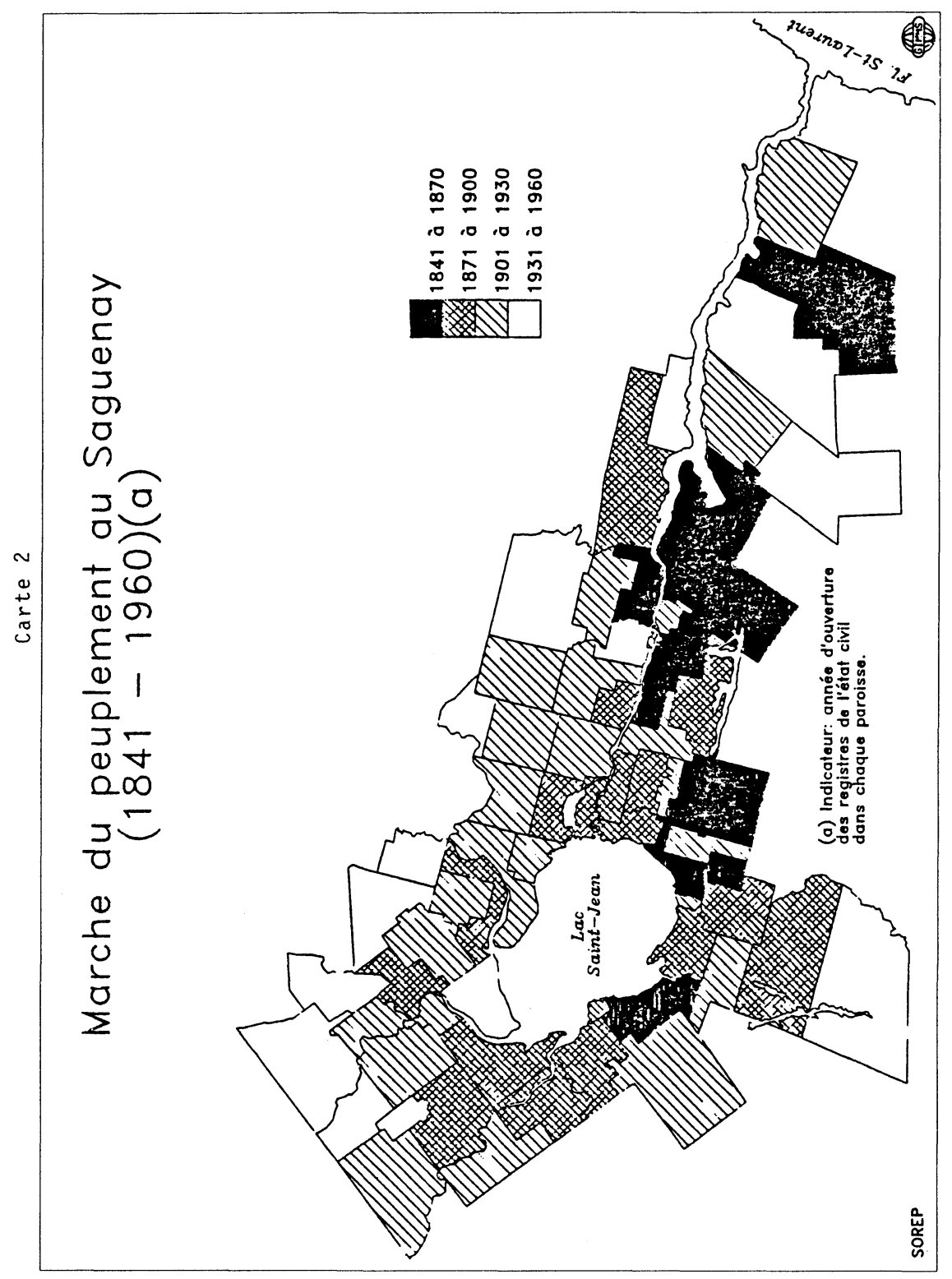




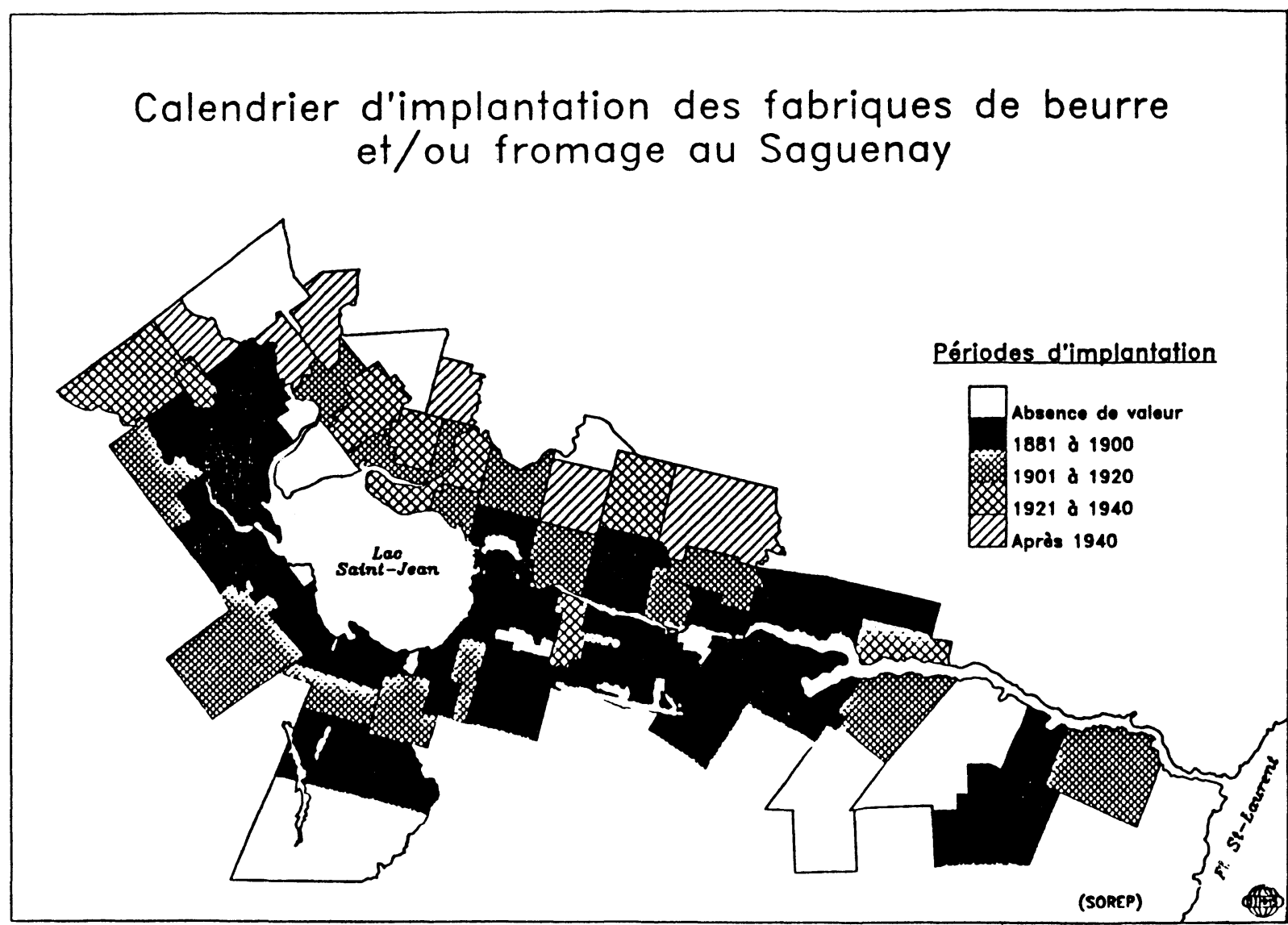

Sources:- Documents de la session du Qućbec. 1883 à 1917. - Annuaire des comiés Chicoutimi el Lac-Saint-Jean. -Fonds "Mgr, Victor Tremblay", "Fromageries a Hebertville" et "Sociélés coopératives agricoles", A.N.Q. 
le Lac-Saint-Jean et le Haut-Saguenay au reste de la province (à partir de 1888 et 1893 respectivement) fut un facteur déterminant dans ce contexte de changement. Il permit à la fois l'exportation plus rapide du fromage et l'importation de produits de base comme la farine ou les graines de semence, que les cultivateurs pouvaient désormais acheter grâce aux revenus de la vente de lait.

Comme on s'en doute cependant, toutes les paroisses ne s'engagèrent pas en même temps ni au même degré dans cette évolution. Il en a résulté, à l'échelle des paroisses et des municipalités, une grande diversité spatiale reflétée dans le calendrier de création des fabriques (Carte 3), la valeur de la machinerie et de l'outillage agricole, la croissance du troupeau laitier et l'introduction des pâturages permanents ${ }^{28}$. Ce phénomène n'a toutefois rien d'exceptionnel, ayant été observé dans d'autres régions du Québec à diverses époques ${ }^{29}$. Dans le cas du Saguenay, on peut faire l'hypothèse que cette stratification était due principalement à l'âge du peuplement et de la mise en valeur des terres, aux caractéristiques climatiques et pédologiques ${ }^{30}$, à la distance par rapport aux zones urbaines et au chemin de fer.

\section{B - La production laitière}

Entre 1891 et 1911, la valeur de la production régionale de fromage et de beurre est passée de $59000 \$$ à $710000 \$$, ce qui atteste la vigueur de la reconversion intervenue ${ }^{31}$. Par ailleurs, notons que jusqu'en 1942, le fromage représentait plus de $80 \%$ de l'ensemble des ventes. Dans certaines fabriques, on faisait un peu de beurre au printemps seulement, à ce moment de l'année où la qualité du lait était médiocre. Même après 1900, alors que les problèmes de conservation étaient à peu près réglés et que la demande de beurre était forte, le Saguenay ne suivit pas la tendance québécoise, s'en tenant à son orientation initiale.

28 Sur chacun de ces points, on trouvera des aperçus statistiques et cartographiques détaillés dans G. Bouchard, R. Thibeault, op. cit.; R. Thibeault, G. Bouchard, op. cit.

29 S. Courville, «Le marché des «subsistances». L'exemple de la plaine de Montréal au début des années 1830: une perspective géographique», Revue d'histoire de l'Amérique française, 42,2 (automne 1988): 193-241; J.-M. Bernard, «Observations sur sept municipalités d'agriculture prospère du Bas-du-Fleuve», Recherches sociographiques, 8,1 (1967): 49-67; G. Fortin, La fin d'un règne (Montréal, Hurtubise HMH, 1971), chapitre 2.

30 M. Gauthier, L'agriculture au Lac-Saint-Jean (province de Québec). Etude géographique thèse de M.A. (géographie), Université du Québec à Chicoutimi, 1975, $331 \mathrm{p}$.

31 D'après les recensements canadiens. Voir M. Saint-Hilaire, Initiation à l' histoire régionale, II: L'industrialisation (Québec et Jonquière, Ministère de l'Éducation (Direction générale des moyens d'enseignement) et Cégep de Jonquière, 1983), $30 \mathrm{p}$. Idéalement, pour tenir compte de l'inflation, il aurait été préférable d'exprimer cette hausse en kilos de fromage et de beurre produits mais il n'est pas possible d'opérer la conversion pour les années considérées. 
Selon les normes du temps, les fromageries saguenayennes étaient nombreuses - ordinairement une par rang, parfois davantage - et de petite taille: en 1925-1930 par exemple, on comptait de 15 à 20 «patrons» ${ }^{32}$ par fabrique (ce qui ne représentait que la moitié environ de la moyenne provinciale) et une dizaine de vaches par patron. Pour le début du $\mathrm{XX}^{\mathrm{e}}$ siècle, à la lumière de toutes les données disponibles, on peut estimer à $20 \$$ environ le revenu annuel d'une vache laitière, une fois enlevée la part du fromager (variable, entre $12 \%$ et $18 \%$ ). Compte tenu de la taille moyenne du troupeau à cette époque (de 6 à 7 vaches au maximum), il faut compter un revenu brut annuel de l'ordre de $120 \$-130 \$$ pour le producteur. Sur la base, encore une fois, de toutes les indications que nous avons pu retirer des sources utilisées (qualitatives aussi bien que quantitatives), on peut affirmer que très fréquemment, cette entrée d'argent ne représentait pas plus du tiers du revenu total de la famille paysanne ${ }^{33}$.

\section{C - Les signes de stagnation}

Une fois passée la période d'implantation, l'industrie laitière saguenayenne a affiché pendant au moins une trentaine d'années (soit entre le début du siècle et la Deuxième Guerre mondiale) une inertie qui s'est manifestée de diverses façons: dans les rendements, le fonctionnement des fabriques, les structures de la production, le troupeau laitier, l'équipement agricole. Entre 1901 et 1941, les rendements de cultures comme le foin et l'avoine furent très erratiques (celui du foin surtout, qui déclina régulièrement à partir de 1911). Quant au rendement annuel des vaches laitières, il fut presque stable sur toute la période, oscillant entre 900 et 1300 litres de lait par vache ${ }^{34}$. Quant aux fabriques, leurs structures ont très peu évolué. Le nombre de patrons $(\mathrm{N}=20-25)$ et de vaches (une dizaine) par fabrique est demeuré presque inchangé durant ces quatre décennies ${ }^{35}$. En fait, dès la fin du $\mathrm{XIX}^{\mathrm{e}}$ siècle, les réformateurs (cf. infra) se plaignaient de ce que les fabriques étaient trop nombreuses, trop petites, sous-équipées et mal-

32 On désignait ainsi les producteurs qui livraient leur lait à une fabrique et s'en partageaient les revenus avec le propriétaire-fromager.

33 Nous tenons compte ici de l'ensemble des revenus d'appoint issus de la pluriactivité (voir G. Bouchard, "Co-intégration et reproduction...», loc. cit., 293ss).

34 D'après les Annuaires statistiques du Québec (données produites par Robert Lavoie dans le cadre d'un mémoire de maîtrise en histoire, sous la direction de l'auteur). Il faut ici porter attention à la tendance affichée durant la période plus qu'aux chiffres absolus de production. Ceux-ci varient d'une source à l'autre, mais non l'allure de la courbe. Ainsi, d'après les recensements canadiens, le rendement annuel serait passé de 1400 à 1975 litres entre 1911 et 1941.

35 R. Thibeault, op. cit., chapitre 8. 
propres $^{36}$. La qualité et le prix du fromage en souffraient, mais il allait s'écouler de nombreuses années avant que les producteurs ne soient sensibilisés à ce problème. Ainsi, comme les fabriques étaient fermées le dimanche, la traite du samedi soir séjournait plus de 36 heures dans des puits domestiques. Malgré les courtes distances séparant la plupart des fermes de la fabrique, le transport du lait s'effectuait dans des conditions précaires eu égard à la conservation. Par ailleurs, c'était très souvent les enfants qui trayaient les vaches, dont le pis n'était pas lavé. Les vaisseaux et bidons étaient mal entretenus et, le lait étant payé au poids sans tenir compte de la teneur en gras $^{37}$, il n'y avait guère d'incitation pour le producteur à en augmenter la qualité. Encore en 1930-1935, l'ensemble des fromageries n'était en exploitation guère plus de six mois par année. Ce n'est pas avant le milieu du siècle en effet que, notamment grâce à une meilleure alimentation, le troupeau laitier put soutenir une production progressivement étendue à toute l'année.

Il y avait des exceptions bien sûr, et la presse locale ne manquait pas de souligner les performances de ces quelques producteurs d'élite: Firmin Paradis de Bagotville, Adhémar Gagné de Laterrière, parmi d'autres. Mais les statistiques à l'échelle de la région font entendre un autre discours. D'après les données des recensements canadiens, de $465068 \mathrm{hl}$ en 1911, la production totale de lait atteignit un sommet en 1921 avec $710818 \mathrm{hl}$. Elle déclina ensuite à $688223 \mathrm{hl}$ après la Crise. La hausse était due à une augmentation non pas de la productivité mais du nombre des fabriques, et elle reflétait essentiellement l'extension du cheptel laitier. Par ailleurs, celle-ci résultait surtout de l'accroissement du nombre des exploitations, consécutif à la poursuite de la colonisation ${ }^{38}$. En fait, les productions de la ferme fluctuaient selon la conjoncture et même les périodes les plus favorables - par exemple 1914-1918 - n'entraînaient que des réaménagements quantitatifs: augmentation du temps de travail, extension des surfaces

36 Voir par exemple Le Progrès du Saguenay, ler mars 1894, 8 octobre 1897.

37 C'est après 1924 seulement que le gouvernement provincial légiféra en ce sens, obligeant ainsi les producteurs à plus de rigueur. À partir de ce moment, une vieille pratique consistant à ajouter de l'eau dans le lait fut progressivement éliminée (sur ce sujet, voir notamment D. Thibault, Nos beurreries et fromageries d'autrefois (S.1., Éditions du Bien public, 1974), 41 p. Au sujet de la piètre qualité du fromage, voir aussi R. Dupré, «Regulating the Quebec Dairy Industry, 1905-1921: Peeling Off the Joseph Label», The Journal of Economic History, 50,2 (juin 1990): 339-348.

38 La dimension des exploitations varia peu entre 1901 et 1931 mais leur nombre s'accrût jusqu'en 1941, tout comme les effectifs de la population rurale (en chiffres absolus, bien sûr). 
cultivées $^{39}$. On connaît par ailleurs de nombreux exemples de sousproduction pure et simple touchant non seulement le lait mais aussi les cultures elles-mêmes ${ }^{40}$.

Deux autres indicateurs témoignent d'une relative inertie de l'exploitation. C'est d'abord l'évolution du troupeau laitier, dont la taille demeura remarquablement stable entre 1910 et 1932, le nombre moyen de vaches par patron passant de 8 à $10^{41}$. C'est ensuite l'évolution de la technologie et de l'équipement agricole. D'après une étude consacrée à ce sujet ${ }^{42}$, on relève a) d'importants changements au tournant du siècle, $b$ ) suivis d'une sorte de plateau s'étendant sur trois décennies environ, puis c) une reprise spectaculaire des transformations dans le sens de la mécanisation et de la rationalisation des procédés, cette dernière étape étant caractérisée principalement par l'extension du cycle annuel de lactation, l'utilisation des engrais chimiques et l'introduction du tracteur, de la trayeuse et de la moissonneuse-batteuse. Ces deux «démarrages» entrecoupés d'une longue période de stagnation concordent parfaitement avec les autres repères déjà présentés ici. Le cas très particulier du silo est tout à fait représentatif de cette périodisation. Apparu sur quelques fermes saguenayennes entre 1890 et 1900 , sa diffusion fut ensuite arrêtée, pour reprendre beaucoup plus tard, vers 1940-1950. La plupart des cultivateurs croyaient que cet équipement était trop sophistiqué pour le Saguenay où la saison de végétation est bien courte. Mais surtout, l'investissement exigé par sa construction ne leur paraissait pas justifié, compte tenu du type d'agriculture qu'ils entendaient pratiquer: parmi d'autres exemples, le rejet du silo exprimait le refus des transformations structurelles qui auraient fait entrer l'exploitation paysanne dans le système de production capitaliste. Le même phénomène est illustré par la sous-production chronique des volailles ou par les lenteurs qui ont caractérisé l'introduction de fourrages plus spécialisés comme la luzerne ou la lentille.

39 G. Bouchard et R. Thibeault, «Change and Continuity in the Saguenay Agriculture: the Evolution of Productions and Yields (1852-1971)», à paraître dans D. H. Akenson, dir., Canadian Papers in Rural History, 8 (1991).

40 Ainsi, des entrepreneurs forestiers qui se plaignent de devoir s'approvisionner en avoine dans l'ouest canadien (voir par exemple I. Pelletier, «Hébertville se raconte (entrevue par N. Perron)», Saguenayensia, 30,2 (avril-juin 1988): 22-25).

41 D’après les Annuaires statistiques du Québec; données (non publiées) compilées par Robert Lavoie.

42 G. Bouchard, «L'agriculture saguenayenne...», op. cit. 


\section{D - Spécificités saguenayennes... ou québécoises?}

Avant d'aller plus loin, il convient de situer ces traits dans une perspective plus large afin de déterminer dans quelle mesure les éléments de stagnation observés étaient exclusifs au Saguenay. Nous disposons à cette fin de quelques indicateurs fiables à l'aide desquels il est possible de proposer un aperçu assez précis, compte tenu de l'état présent de la recherche sur l'agriculture québécoise ${ }^{43}$. Il importe d'abord de souligner que, sur plusieurs points importants, la trame de l'évolution agricole au Saguenay reproduit exactement ou suit d'assez près celle de l'ensemble du Québec, ou celle d'un comté-témoin comme Champlain en Mauricie, qui a déjà fait l'objet d'une étude détaillée ${ }^{44}$. C'est le cas par exemple pour le calendrier d'introduction du tracteur, de la moissonneuse-batteuse et de la trayeuse mécanique; c'est aussi le cas pour le rendement du seigle, du foin et des vaches laitière $\mathrm{s}^{45}$ tout comme pour la durée annuelle de production des fromageries. À ce propos, il est utile de rappeler que plusieurs régions d'industrie laitière au Québec (à en juger du moins par les données agrégées pour la province) montrent les mêmes symptômes d'inertie que le Saguenay. À l'appui de cet énoncé, on peut citer diverses études attestant une stagnation des rendements des cultures ${ }^{46}$, des phénomènes de sous-production généralisée ${ }^{47}$, une organisation déficiente des fabriques de beurre et de fromage ${ }^{48}$, etc. Sous tous ces rapports, les performances québécoises étaient dépassées par celles de l'Ontario, qu'il s'agisse de la production annuelle par fromagerie ${ }^{49}$, de la production annuelle des vaches laitières ${ }^{50}$, de la productivité du travail agricole $^{51}$ ou du rythme de la mécanisation ${ }^{52}$.

Ces données invitent à relativiser les «tares» saguenayennes et suggèrent que les facteurs structurels auxquels il faut les imputer

43 En effet, il n'est pas possible actuellement d'effectuer une comparaison à l'échelle des régions. C'est cependant l'objectif d'un projet de recherche en cours à SOREP et qui n'en est pour l'instant qu'au stade de la collecte des données (voir à ce sujet le Rapport annuel 198990 du Centre SOREP, au projet VALIN).

44 N. Séguin, «L'agriculture de la Mauricie et du Québec, 1850-1950», Revue d' histoire de l'Amérique française, 35,4 (mars 1982): 537-563.

45 G. Bouchard, R. Thibeault, «Change and Continuity...», loc. cit.

46 J.-P. Wampach, loc. cit.

47 L. Gérin, «La famille canadienne-française, sa force, ses faiblesses», M. Rioux et Y. Martin, La société canadienne-française (Montréal, Hurtubise HMH, 1971), 45-67.

48 N. Perron, «Genèse des activités...», loc. cit.

49 Ibid.

50 G. Bouchard, R. Thibeault, "Change and Continuity...», loc. cit.

51 J.-P. Wampach, loc. cit.

52 C. Blouin, «La mécanisation de l'agriculture entre 1830-1890», N. Séguin, Agriculture..., 93-111. 
n'étaient pas non plus exclusifs au Saguenay. Par contre, il est indéniable que cette région accusait aussi un recul par rapport aux moyennes québécoises, sur des points comme le calendrier d'introduction des engrais chimiques, le rendement de l'avoine et de l'orge, l'adaptation au marché du beurre, la dimension des fabriques. Selon l'hypothèse qui fonde le présent article, ces traits, tout comme les autres, seraient pour une bonne part tributaires de structures sociales et culturelles. Mais cette inférence est prématurée, à ce stade-ci de notre réflexion; il faut encore montrer que, dès la fin du XIX ${ }^{\mathrm{e}}$ siècle, l'économie paysanne saguenayenne bénéficiait des conditions objectives nécessaires à sa croissance.

\section{3 - LES FACTEURS FAVORABLES À LA CROISSANCE}

Par conditions objectives, il faut entendre ici l'ensemble des facteurs (physiques, techniques, économiques, institutionnels) en l'absence desquels le capitalisme agraire aurait eu peu de chance de réussir, quel que soit l'état d'esprit des exploitants. Or, nous voulons démontrer que tous ces facteurs étaient présents au Saguenay dès 1890-1900 et qu'on ne saurait rendre compte par ce biais des éléments de stagnation décrits plus haut.

\section{A - Facteurs physiques et techniques}

Les sols et surtout le climat saguenayen, comme dans plusieurs régions du Québec, se prêtaient particulièrement bien à l'élevage laitier. Mieux que les diverses espèces de céréales disponibles à l'époque, le fourrage résistait à la fois aux basses températures de début et de fin d'été, aux chaleurs de juillet parfois accompagnées de sécheresse, ainsi qu'à l'humidité causée par un faible ensoleillement et par d'abondantes précipitations. Les sols, même mal préparés, se prêtaient aussi très bien aux cultures fourragères. Pour ce qui est de la valeur de la race bovine sur les fermes du Saguenay, on la jugeait en général de moyenne à médiocre; mais compte tenu de la situation générale de l'élevage au Québec à cette époque, compte tenu aussi de la conjoncture commerciale très favorable, ce facteur aurait pu aisément être compensé par une meilleure alimentation et par des soins plus attentifs.

De même, sur le plan technique, la spécialisation dans l'industrie laitière ne représentait guère de difficultés pour l'exploitant. Elle supposait une extension de cultures fourragères plutôt robustes, demandant peu de soins, de même qu'un abandon de céréales secondaires comme l'orge et le seigle. Quant au blé, il était de toute manière condamné à cause de la rigueur du climat, comme le montrait 
la succession des mauvaises récoltes. Pour le reste, le travail de la ferme demeurait pratiquement inchangé; à court terme, il exigeait peu d'équipement supplémentaire (le silo n'était pas une nécessité) et le fardeau des tâches se trouvait même soulagé d'une partie du battage à l'automne. En fait, l'investissement technologique entraîné par l'industrie laitière était concentré sur la fabrique; mais ceci était l'affaire du fromager et l'habitant ne s'en mêlait pas directement.

Le principal empêchement d'ordre physique aurait pu résider dans la distance séparant le Saguenay de Montréal, point principal d'expédition pour l'Angleterre. Mais pour diverses raisons, on ne peut y voir un obstacle sérieux à la compétitivité des producteurs et à la rentabilité de leurs opérations. D'abord, comme il a été signalé plus haut, dès 1893, l'ensemble de la région (de Chicoutimi à Roberval) était relié à Québec et à Montréal par chemin de fer. Certes, le transport ferroviaire comportait un coût mais tout indique qu'il représentait un inconvénient relativement mineur. Jusqu'en 1892-1895, il arrivait souvent que le fromage du Saguenay soit vendu à un prix inférieur à celui de Montréal. Mais les producteurs et autres intervenants (auxquels le Progrès du Saguenay faisait abondamment écho) en attribuaient la cause à la situation de monopole dont jouissaient les acheteurs montréalais, et non pas aux coûts de transport. En effet, ces acheteurs déléguaient un représentant qui traitait individuellement avec les fabriques, leur offrant un prix unique, non négociable. Cette situation fut vite éliminée lorsque les producteurs s'unirent pour former des syndicats de vente (voir infra). À partir de ce moment, les producteurs obtenaient souvent des prix identiques à ceux du marché montréalais ou à peu de chose près. Dans le cas contraire, l'écart de prix était surtout imputable à la qualité inférieure du produit et, en définitive, au caractère trop artisanal des fabriques. Ces deux facteurs étaient en effet étroitement liés, la qualité du fromage augmentant avec le volume de la production ${ }^{53}$. Quant au coût de transport proprement dit entre le lieu de la fabrique et Montréal, l'enquête Canada et Québec de 1931-1932 l'établissait en moyenne à 3,4\% du prix officiel (soit, pour l'année 1931, 0,383 cent sur un prix moyen de 11,12 cents la livre $)^{54}$. D'après les indications fournies par l'enquête, on peut estimer à $5 \%$ ou $6 \%$ environ le coût de transport pour les seules

53 Sur ce point, les témoignages d'experts abondent. Voir, en particulier, les résultats d'une enquête détaillée sur 120 fabriques, réalisée en 1931-1932 par les soins des ministères de l'Agriculture du Canada et du Québec (Canada et Québec, Ministère de l'Agriculture, Analyse économique des opérations des fromageries dans le Québec (Ottawa et Québec, 1933), 39 p.).

54 Des 120 fabriques étudiées, 60 étaient situées au Saguenay et les autres au sud-ouest de Québec. Les résultats ne sont pas donnés par région. 
fabriques saguenayennes, ce qui représente en définitive un fardeau bien supportable, surtout si l'on tient compte de la rationalité économique propre à ces exploitants - nous y reviendrons. Un dernier point vient confirmer cet énoncé: dans toutes les archives et la littérature locales consultées sur l'industrie laitière, nous n'avons relevé aucune plainte ni même aucune référence en rapport avec le problème qu'auraient pu constituer les coûts de transport.

\section{B - Animation et information}

Sur un autre plan, dès la décennie 1890-1900, les informations techniques et les encouragements étaient prodigués en abondance aux cultivateurs saguenayens. De toutes parts, on tentait de leur démontrer les avantages de l'élevage laitier. Cette propagande était le fait de réformateurs qui se recrutaient d'abord parmi les professions libérales et les élites laïques: l'avocat L. de Gonzague Belley de Chicoutimi, qui en faisait un sujet de discours patriotiques; le juge Thomas-Louis Bergeron de Chambord, qui s'adonnait lui-même à l'agriculture; le député Jos. Girard de Saint-Gédéon qui, en 1893, dirigeait une délégation de 130 producteurs à Québec pour participer au premier congrès provincial des cultivateurs; le notaire P.-H. Dumais, de Chambord également, qui fut un pionnier de l'ensilage; et Avila Charbonneau, le premier agronome permanent au Saguenay, artisan des jardins scolaires ${ }^{55}$. Mais de ce côté, la palme revient sans contredit à Joseph-Dominique Guay de Chicoutimi, à l'époque où il était propriétaire-directeur de l'hebdomadaire Le Progrès du Saguenay. Entre 1895 et 1918, il fut tour à tour, et presque sans interruption, directeur, vice-président et président de la Société d'agriculture du comté. Â peu près durant la même période, il occupa des fonctions analogues au sein du cercle agricole de Chicoutimi. À titre de conférencier agricole, il a visité à plus d'une reprise toutes les paroisses du Haut-Saguenay, prêchant en faveur de l'ensilage, des engrais, d'une meilleure alimentation des vaches, d'une extension des cultures fourragères. Il exploitait lui-même avec succès, à proximité de la ville, une ferme réputée qui lui valut en 1899 une médaille d'argent au concours provincial du Mérite agricole. Il exposait dans la vitrine de son imprimerie à Chicoutimi un échantillon des produits de ses champs et se servait abondamment de son hebdomadaire pour diffuser ses idées. Il fut ainsi l'un des premiers en 1893 à prôner l'évaluation du lait sur la base de sa teneur en gras et non seulement de son poids. Il fut

55 Voir Le Progrès du Saguenay, 19 novembre 1891, 9 février 1893, 12 avril 1894, ler avril 1920. 
également très actif dans la création des syndicats de vente de fromage.

Les prêtres et curés apportèrent aussi une contribution importante dans cette campagne en faveur d'une agriculture plus rationnelle, axée sur l'industrie laitière. À Saint-Charles, au nord-ouest de Jonquière, c'est le curé Larouche qui, en 1893, introduisait l'usage des graines de semence et des fourrages verts. À la même époque, dans une autre paroisse du Lac-Saint-Jean (Lac-Bouchette), le curé J.-F. Roy faisait l'essai du sarrasin comme engrais. À Laterrière, près de Chicoutimi, c'est le curé F.-X. Delage qui faisait l'achat des graines de semence pour l'ensemble de sa paroisse. Ailleurs, des curés-agriculteurs annonçaient fièrement en chaire les rendements de leurs cultures et de leurs vaches laitières ${ }^{56}$.

Parallèlement, des conférenciers gouvernementaux sillonnaient régulièrement les paroisses, incitant les habitants à imiter ce qu'on tenait à l'époque pour des fermes-modèles: celle des prêtres du Séminaire de Chicoutimi où l'on pratiquait l'ensilage et où le visiteur pouvait admirer un coupe-paille mû par un cheval; celle des Ursulines de Roberval, qui mérita en 1894 le premier prix du Mérite agricole et dont la responsable portait le titre d'agronome; celles aussi des Pères Trappistes à Mistassini, des Sœurs de l'Hôpital à Chicoutimi; enfin celles créés par des entrepreneurs forestiers pour l'approvisionnement de leurs chantiers (Price à Grande-Baie, Beemer à Roberval, Scott à Mistassini...). Mentionnons enfin, toujours au chapitre de l'animation, les expositions et concours agricoles qui, annuellement et dans plusieurs municipalités de la région, attiraient l'attention des habitants sur les nouveaux procédés et équipements. Ici, comme dans l'organisation de l'enseignement et des conférences agricoles, l'apport des agents gouvernementaux était prédominant. Leur action s'exerçait aussi de bien d'autres façons: par les subventions versées aux sociétés d'agriculture et aux cercles agricoles, les primes offertes aux beurreries et aux éleveurs les plus méritants, l'aide financière à la construction de silos, le soutien à la publication des journaux agricoles, etc.

Dans l'ensemble cependant, toutes ces activités ne touchaient qu'une minorité d'agriculteurs; la masse ne suivait pas, s'en tenant à sa routine. Que de fois, par exemple, a-t-on déploré que les conférenciers les plus éclairés se soient produits devant des salles presque vides.

56 Sur ce sujet, voir par exemple Le Progrès du Saguenay, 21 mars 1889, 29 décembre 1892, 28 septembre 1893, 11 janvier 1894, 19 avril 1894, 28 janvier 1904, etc. 


\section{C - Encadrement institutionnel}

Dès la fin du XIX ${ }^{\mathrm{e}}$ siècle aussi, des structures d'encadrement étaient en place pour assister et guider les producteurs dans leur reconversion. Ces structures consistaient, bien sûr, dans des institutions d'enseignement comme l'École d'industrie laitière de SaintHyacinthe (près de Montréal) fréquentée par quelques cultivateurs de la région, comme l'École ménagère des Ursulines de Roberval et les Cercles de fermières qui en furent le prolongement, ou encore les deux Sociétés d'agriculture (celle du Haut-Saguenay et celle du Lac-SaintJean $)^{57}$. Mais l'instance qui était le plus près des cultivateurs était assurément le cercle agricole. Cette formule d'association qui œuvrait à l'échelle paroissiale est apparue en 1862 au Québec. Fédérés une première fois en 1875, les cercles reçurent leur constitution en 1886 de l'assemblée des évêques du Québec. En vertu de leurs règlements, les cercles étaient placés sous la direction immédiate du curé de la paroisse et sous le patronage de l'évêque du diocèse. Ils devaient aussi poursuivre des objectifs tant moraux que professionnels ${ }^{58}$. Mais la pratique en décida autrement.

À partir de 1892, les créations de cercles furent nombreuses au Saguenay (le premier cercle étant celui du rang Saint-Pierre de Chicoutimi, en novembre 1892, à l'initiative de J.-D. Guay). Dès la fin de 1893, il existait 24 cercles dans la région ${ }^{59}$, financés surtout par le gouvernement provincial. Quelques-uns, très dynamiques, exerçaient une réelle influence sur les pratiques agraires. C'était le cas par exemple des cercles de Saint-Gédéon et d'Hébertville, dont les archives ont été conservées ${ }^{60}$. Le premier, comme tous les autres cercles, effectuait des achats collectifs de graines de semence et d'équipements, accueillait régulièrement des conférenciers du gouvernement et organisait un concours annuel. Mais il était aussi un véritable lieu d'animation et d'échange. Le cercle encourageait les essais de nouvelles espèces ou de nouveaux procédés et distribuait des prix (en argent) aux producteurs les plus efficaces. Les assemblées donnaient lieu très souvent à de vives discussions sur des points très techniques. Chacun y faisait rapport de ses essais et résultats. On y commentait des articles du Journal d'Agriculture, on se fixait un programme, des objectifs annuels de production; on vota même un salaire au meilleur

57 Sur ces sociétés d'agriculture, voir N. Perron, «Une agriculture naissante...», loc. cit., 174-176.

58 Sur ce sujet, F. Létourneau, op. cit.; J. Hamelin, Y. Roby, op. cit., 189ss.

59 D'après Le Progrès du Saguenay, 3 et 10 novembre 1892, 12 octobre 1893.

60 ANQC, Fonds de la Société historique du Saguenay, Documents nos 48 et 494. 
cultivateur de la paroisse pour qu'il donne des leçons aux membres deux fois la semaine, etc.

À peu de chose près, le cercle d'Hébertville reproduisait le modèle de Saint-Gédéon. Mais il ne fait aucun doute que ces deux cercles faisaient exception, tout comme ceux de Laterrière, Chicoutimi et Bagotville. Pour le reste, les cercles vivotaient, ressuscitant une fois par année (au printemps) pour l'achat collectif de graines de semences et d'animaux. Tous les témoignages concordent sur ce point: dans la majorité des cas, l'effervescence des premières années a fait place à l'apathie ${ }^{61}$. Le principal objectif visé, qui était d'entretenir une volonté de changement à long terme parmi les cultivateurs, n'a pas été atteint.

\section{D - Mise en marché}

On peut montrer par ailleurs que dès le début du $\mathrm{XX}^{\mathrm{e}}$ siècle, tout un appareil de mise en marché était en place, dont l'utilisation dépendait surtout de la volonté de l'habitant. D'abord, dès l'automne 1888 , les cultivateurs de Chicoutimi se souciaient de mieux organiser la vente de leurs produits en déléguant un chargé d'affaires à Montréal. Deux ans plus tard, des producteurs réunis à Bagotville créaient le Syndicat des fromagers du comté de Chicoutimi, dont le but était de contourner les courtiers de Montréal en vendant directement le fromage en Angleterre - cette initiative faisait écho à une politique mise en œuvre par le Conseil d'Agriculture de la province de Québec ${ }^{62}$. Les choses n'en sont pas restées là. À l'initiative d'un autre groupe de producteurs, à partir de l'automne 1892, le fromage fut transigé à Chicoutimi à tous les quinze jours ou à tous les mois. Parallèlement, des syndicats de producteurs furent mis sur pied dans le Haut-Saguenay et au Lac-Saint-Jean, conformément au programme de la Société d'industrie laitière de la province de Québec ${ }^{63}$. Avec l'appui de divers organismes (dont la Banque Nationale), d'autres syndicats concurrents virent aussi le jour dans les années suivantes, essentiellement avec les mêmes buts: briser le monopole des acheteurs en vendant collectivement au plus offrant, informer et protéger les producteurs $^{64}$.

61 Le cercle d'Hébertville lui-même, souvent donné en exemple pour son dynamisme, a dû en 1894 assigner à un membre la tâche de «maintenir le bon ordre pendant les assemblées avec pouvoir de faire absenter tous ceux qui ne voudront pas se tenir sages et bien écouter...». (Assemblée du 13 avril 1894, Doc. 494, fol. 91).

62 Le Progrès du Saguenay, 13 septembre 1888, 30 octobre 1890, 6 mai 1891.

63 Le Progrès du Saguenay, 13 avril 1892, 27 octobre 1892, 28 février 1893, 29 juin 1893, ler mars 1894, 22 mars 1894, etc.

64 Le Progrès du Saguenay, 20 et 27 juin 1895, 8 octobre 1896, 31 mai 1900, 27 mars 1902, 16 juin 1904, 15 mars 1906. 
Mais en plus de procurer des revenus plus élevés aux cultivateurs, ces premiers syndicats visaient aussi à éliminer les petits fabricants mal organisés et à hausser la qualité du produit. Il semble toutefois que ce deuxième objectif ait été abandonné en cours de route. À en juger par les chroniques du Progrès du Saguenay entre 1910 et 1925, les syndicats furent en quelque sorte victimes de leur succès; ils ne surent pas résister à une extension de leur membership qui fit bientôt une large place aux producteurs de second ordre. Ces derniers trouvaient ainsi dans la formule associative une protection facile qui les dispensait de réformer leur entreprise.

Quant aux coopératives agricoles qui furent créées à travers le Québec à partir de 1909, elles auraient pu être - comme le souhaitait le gouvernement - un puissant véhicule de consolidation et de transformation de l'agriculture. Mais les cultivateurs saguenayens boudèrent cette première tentative pour mettre le principe associatif au service de la modernisation des fermes. Même lorsque l'idée commença à connaître du succès entre 1920 et 1930, c'est dans le cadre paroissial uniquement qu'elle se concrétisa et c'est avec peine qu'à partir de la Deuxième Guerre mondiale des coopératives d'envergure régionale ou multi-paroissiale purent s'implanter ${ }^{65}$. Mais ici encore une fois, le Saguenay ne fut pas un cas isolé; une résistance semblable se manifesta en effet dans d'autres régions du Québec, telles les Laurentides, l'Outaouais, le Bas-Saint-Laurent.

\section{4 - LA CO-INTÉGRATION}

Ce dernier épisode illustre parfaitement l'argument du présent essai: au-delà d'un certain seuil, la spécialisation et la commercialisation de l'agriculture mettaient en péril des pratiques de travail, des objectifs socio-économiques ainsi qu'un mode de vie réfractaires au système capitaliste et aux formes socio-culturelles qui lui étaient associées. En lui-même, ce simple fait serait banal — on connaît de multiples exemples de telles résistances - s'il ne mettait en cause une région qui, de tant de façons, était déjà intégrée à la société capitaliste et réunissait toutes les conditions favorables à cet égard. Notre analyse et certaines données présentées ailleurs ${ }^{66}$ établissent en effet que, dès le tournant du siècle, la technologie requise existait, le savoir technique était largement diffusé et les conditions climatiques tout à fait

65 Sur ce sujet, voir les résultats d'une étude récente conduite à SOREP (N. Perron, "L'évolution des institutions...», loc. cit.).

66 G. Bouchard, «La dynamique communautaire...», loc. cit.; «Sur la reproduction familiale en milieu rural: systèmes ouverts et systèmes clos», Recherches sociographiques, 28,23 (1987): 229-251; «Co-intégration et reproduction...», loc. cit. 
appropriées. Au nombre des facteurs favorables, il faut également citer la demande soutenue pour les produits laitiers, le régime de propriété qui faisait de l'habitant un exploitant autonome, la dimension des exploitations, la main-d'œuvre nombreuse, des voies de communication ferroviaire et maritime, des institutions de mise en marché et d'encadrement, des mesures d'incitations. Or il faut insister sur le fait que les agriculteurs n'ont pas vraiment tourné le dos à la nouvelle option que représentait l'industrie laitière; plutôt, ils l'ont exploitée d'une manière qui paraît peu rationnelle, à première vue du moins. C'est précisément là le propre de la co-intégration: entretenir avec la société ou l'économie extra-régionale des liens nombreux et persistants, non pour s'y intégrer mais pour l'utiliser afin de consolider et de reproduire des orientations collectives radicalement différentes, sinon opposées.

Rappelons que la région du Saguenay ne fut ouverte qu'en 1840 au peuplement blanc et que la colonisation s'y est poursuivie pendant un siècle. Dans ce contexte d'inorganisation institutionnelle, le groupe familial a longtemps joué un rôle prioritaire dans l'appropriation et la mise en valeur de la terre. En fait, la reproduction et la fixation de la société rurale ont dépendu largement du mode de reproduction de la famille. À la faveur de la colonisation, un système original ${ }^{67} \mathrm{de}$ transmission des avoirs s'est mis en place, qui visait à établir le maximum d'enfants sur des terres - soit les trois quarts des garçons et filles environ, parmi les couples formés avant 1911. La fécondité élevée à la fois nécessitait l'expansion physique des patrimoines et la rendait possible en assurant une main-d'œuvre nombreuse pour les défrichements ${ }^{68}$. Ce mode de reproduction familiale, comme il a été dit plus haut, permettait de perpétuer de vieux traits socio-culturels dans un espace social marginalisé. On aurait tort pourtant de considérer cette société comme statique ou passive. Elle était au contraire en croissance rapide et faisait preuve d'un grand dynamisme - le rythme de son accroissement naturel et l'extension de l'écoumène en témoignent - mais ses énergies et ses investissements étaient canalisés suivant des voies qui n'étaient pas celles du système capitaliste.

67 Par rapport aux «vieux pays» d'Europe surtout, car le système saguenayen était semblable à celui qui a prévalu dans de nombreuses régions de peuplement en Amérique du Nord (G. Bouchard, «Les systèmes de transmission des avoirs familiaux et le cycle de la société rurale au Québec, du XVII ${ }^{e}$ au XX' $X^{e}$ siècle», Histoire sociale/Social History, 16,31 (mai/may 1983): 35-60; «L'historiographie du Québec rural et la problématique nord-américaine avant la Révolution tranquille. Étude d'un refus», Revue d'histoire de l'Amérique française, 44,2 (automne 1990): 199-222.

68 Sur ce sujet, G. Bouchard, «Sur la reproduction familiale...», loc. cit. 
Ce faisant, la société rurale saguenayenne arrivait à préserver une forme d'autonomie assez particulière qui est le lieu d'un paradoxe. Par le biais de la pluriactivité et en mettant à profit le travail des enfants, la famille paysanne satisfaisait à ses besoins en numéraire. Diverses sources d'appoint produisaient, chacune isolément, un revenu médiocre; mais ces revenus combinés assuraient le minimum requis. Pour cette raison, il n'est pas exagéré de dire que la famille réalisait son autonomie à même une multiplicité de dépendances, dont aucune ne pouvait vraiment imposer sa loi. De chacune, elle attendait un revenu modeste mais sûr, sans trop porter attention à la quantité de travail requis; car c'était là une ressource dont elle disposait en abondance.

C'est ce système qui a présidé à la gestion de la production laitière et qui explique ses caractéristiques. Les ventes de lait ne procuraient qu'un revenu parmi d'autres ${ }^{69}$. Sans nécessairement conclure à un refus de changement faisant suite à un choix explicite, rationnellement effectué, il est clair qu'une spécialisation en fonction de cette activité aurait mis en échec le mode traditionnel de reproduction familiale de même que la division du travail, elle-même tributaire de la structure d'autorité dans la famille. C'est à cela qu'il faut rapporter toutes les formes de résistance ou d' «apathie» relevées plus haut: refus de donner suite à l'exemple des fermes-modèles et à l'enseignement des cercles, maintien de procédés désuets et de rendements stagnants, utilisation des syndicats de vente pour protéger les producteurs déficients, retard à exploiter le marché rentable du beurre, attachement à la petite fromagerie de rang sous-équipée et déficiente, alors même que les avantages de la concentration étaient bien établis. Ce dernier point, visible aussi dans la résistance contre les coopératives régionales, est peut-être le plus révélateur. Dans l'esprit de la majorité des cultivateurs, la fromagerie, avec ses revenus faciles, faisait partie de l'équipement collectif local, tout comme le moulin à moudre ou la boutique du forgeron ${ }^{70}$. Sa dimension et son fonctionnement étaient parfaitement articulés aux structures de cette paysannerie: ne menaçant aucunement l'équilibre de la co-intégration, elle lui procurait au contraire un appui additionnel.

C'est sur cet arrière-plan aussi qu'il faut considérer le problème de la mécanisation. Celle-ci s'est intensifiée après la Crise de 1929 mais

69 À part les activités externes proprement dites, l'exploitation elle-même produisait d'autres revenus: vente d'animaux, de peaux, d'avoine, de fourrage, d'oeufs, de bois de chauffage, etc.

70 Ils le disaient eux-mêmes: à l'époque du peuplement, l'existence d'une fromagerie dans un rang était un facteur important d'attraction pour les nouveaux colons. Voir ANQC, Mémoires de vieillards, no 825,1 . 
elle s'était manifestée à partir de la fin du $\mathrm{XIX}^{\mathrm{e}}$ siècle (achats de râteaux, de faucheuses, de batteuses...). Or certains auteurs ont fait valoir que cette première mécanisation représentait une dépense voluptuaire, trahissant surtout le goût de l'habitant pour les machines $^{71}$. Il est vrai que leur introduction ne s'est pas accompagnée d'une hausse proportionnée des rendements et qu'elle a servi en partie à diminuer la charge de travail des paysans. Mais dans plusieurs cas aussi, la mécanisation a permis d'étendre les surfaces cultivées et d'accroître les productions. Quoi qu'il en soit, cette utilisation de la machine était parfaitement conforme à la rationalité de la cointégration: l'exploitant pouvait utiliser sa main-d'œuvre à d'autres travaux (pas nécessairement sur la ferme) et il s'assurait de pouvoir tirer profit des conjonctures favorables, comme il arriva en 1914-1918.

Le cadre d'interprétation ici proposé pour rendre compte des éléments de stagnation de l'agriculture saguenayenne doit encore faire justice de trois hypothèses concurrentes, que nous présentons brièvement. C'est d'abord l'idée que les retards dans le développement de la production laitière seraient imputables au fait qu'elle était peu rentable, sinon carrément déficitaire. À quelques reprises, Le Progrès $d u$ Saguenay a fait écho à cette thèse, chiffres à l'appui ${ }^{72}$. Il est difficile de souscrire à cette stricte comptabilité qui traduit les coûts de production en fonction de leur valeur sur le marché. Car, précisément, ce n'était pas l'univers de référence des agriculteurs qui disposaient de beaucoup d'espace et de main-d'œuvre. Le déficit comptable était ici parfaitement compatible avec une rentabilité de type social, plus en conformité avec les objectifs et la rationalité de ces familles paysannes soucieuses d'abord d'assurer leur reproduction. Il faut aussi repousser l'hypothèse voulant que la récession brutale qui a suivi la Première Guerre mondiale ait inspiré aux exploitants une grande méfiance à l'endroit des mécanismes du marché, ce qui aurait inspiré leurs comportements réfractaires. Nous pensons que cet argument ne vaut que pour les cultivateurs les plus progressistes et les plus audacieux, ceuxlà même qui avaient misé sur une conjoncture très favorable pour accélérer la restructuration de leur exploitation. On sait en effet que la majorité des cultivateurs saguenayens ont tiré profit du marché créé par la guerre en se contentant d'accroître les facteurs de production,

71 R. Blanchard, L'Est du Canada français. «Province de Québec» (Paris et Montréal, Librairie Masson \& Cie et Librairie Beauchemin Limitée, 1935), 2: 128ss; C. Lemelin, «Social Impact...», loc. cit.; H. Miner, "A New Epoch in Rural Quebec», The American Journal of Sociology, 56,1 (1950): 1-10.

72 Voir les livraisons du 15 décembre 1892, 24 janvier 1918, 4 mars 1927. Aussi, N. Perron, «Une agriculture naissante...», loc. cit. 
surtout les superficies cultivées et le temps de travail ${ }^{73}$. Enfin, selon une troisième explication possible, la conversion au capitalisme agraire aurait nécessité des capitaux dont les cultivateurs ne disposaient pas. Sur ce point, tout est question de calendrier. Le raisonnement est plausible dans l'hypothèse d'une conversion très rapide et en profondeur, sans préparation. Mais il ne s'agit pas de cela ici. La stagnation s'est étendue sur une période de plus de trois décennies; c'était amplement suffisant pour réaliser des changements substantiels que les exploitants auraient pu mettre en ouvre progressivement, à un rythme très prudent, au gré d'investissements modestes financés à même les revenus du fromage ou de la pluriactivité. Sur trente-cing ans, l'avance accumulée de cette façon aurait pu être spectaculaire. Il nous paraît clair que c'est la volonté de réforme qui faisait défaut, pour les raisons mentionnées plus haut.

\section{CONCLUSION}

Si tout reposait ainsi sur la co-intégration, il faut encore se demander pourquoi celle-ci s'est mise en place au Saguenay et comment elle a pu s'y maintenir. D'une façon générale, il semble que la cointégration apparaisse à la faveur de segmentations au sein de la société globale, celles-ci donnant lieu à autant de dissociations dans la dynamique des appartenances, de l'intégration et de la reproduction sociale. Ces segmentations peuvent être imputables à des facteurs très divers: des différences religieuses, linguistiques ou ethniques, l'isolement géographique, des disparités économiques et sociales, des écarts dans les rythmes et les modes de développement, etc. Dans le cas particulier du Saguenay, la rupture a été créée principalement par le contexte de la colonisation et les traits qui lui étaient associés: l'éloignement, l'abondance des terres à défricher, l'accès facile à la propriété foncière, le rôle prépondérant du groupe familial (imputable en partie à la pauvreté institutionnelle ambiante), la vigueur des solidarités locales, condition essentielle à la survie matérielle, et enfin le hiatus engendré par la mise en place d'un mode de production très rudimentaire, caractéristique des régions de peuplement. En conséquence, la nouvelle société s'est constituée comme une entité distincte (ou marginale) et elle a utilisé ses relations avec le marché - et notamment le commerce du lait - pour consolider son propre modèle de reproduction et de croissance.

À des degrés divers, ce système put se maintenir pendant toute la durée de la colonisation. Mais, si dans un premier temps, il a permis

73 G. Bouchard, R. Thibeault, «Change and Continuity...», loc. cit.; «Données sur l'évolution de l'industrie laitière», op. cit. 
l'implantation et la reproduction de la population rurale, on peut dire qu'il a par la suite compromis son développement. En fait, les traits du système se sont durcis dans des traditions et des normes de plus en plus inadaptées; par une sorte d'inertie, il a survécu aux conditions qui l'avaient engendré. Il a été finalement vaincu par une conjonction de facteurs, les principaux étant a) la saturation de l'écoumène cultivable, qui mit en échec le vieux mode expansionniste de reproduction familiale, b) l'éclatement de la pluriactivité, consécutive à l'évolution de l'économie capitaliste ${ }^{74}$, c) les progrès de l'urbanisation, qui diffusèrent de nouveaux modèles de consommation à l'échelle du Québec et qui, au Saguenay même, donnèrent naissance à un marché régional de plus en plus rentable et facile à exploiter, d) la pression exercée sur la main-d'œuvre familiale par la croissance des emplois non agricoles permanents et relativement bien rénumérés. On vit ainsi, à partir de 1940-1945, de nombreux signes d'un deuxième démarrage agraire (après celui de 1890-1900) se traduisant notamment par une hausse spectaculaire des rendements, une concentration de la production et une rupture de la pluriactivité. Parallèlement, rendue progressivement à ses finalités strictement économiques, l'agriculture se repliait sur les sols les plus fertiles. La paysannerie cédait au capitalisme, glissant de la co-intégration à l'intégration.

Cet essai prenait pour objet l'évolution de l'économie agricole dans la région du Saguenay. Mais au-delà de ce cadre très circonstancié, il se pourrait que la démarche explicative proposée trouve une aire d'application plus large. En définitive, ce que le modèle de la cointégration voudrait contribuer à éclairer, c'est toute la question des déphasages et discordances qui se creusent entre les segments d'une société globale et qui se perpétuent, assez paradoxalement, grâce à une dynamique de la réciprocité. Or, nous avons vu que, du point de vue du développement de l'industrie laitière, la trame saguenayenne ne faisait pas vraiment exception dans l'ensemble québécois. Cette donnée ouvre des perspectives nouvelles à notre démarche.

74 Les rapports de co-intégration devinrent de moins en moins fonctionnels. Par exemple, après la Crise, les entreprises forestières étendirent les travaux d'abattage sur toute l'année. De saisonnier, l'emploi de bûcheron devint permanent, privant ainsi les familles paysannes d'un important revenu d'appoint. 